\title{
LIE DERIVATIONS ON TRIVIAL EXTENSION ALGEBRAS
}

\author{
Amir Hosein Mokhtari, Fahimeh Moafian, \\ HAMID REZA EBRAHIMI VISHKI
}

\begin{abstract}
In this paper we provide some conditions under which a Lie derivation on a trivial extension algebra is proper, that is, it can be expressed as a sum of a derivation and a center valued map vanishing at commutators. We then apply our results for triangular algebras. Some illuminating examples are also included.
\end{abstract}

\section{Introduction}

Let $\mathfrak{A}$ be a unital algebra (over a commutative unital ring $\mathbf{R}$ ) and $\mathfrak{X}$ be an $\mathfrak{A}$-bimodule. A linear mapping $\mathcal{D}$ from $\mathfrak{A}$ into $\mathfrak{X}$ is said to be a derivation if

$$
\mathcal{D}(a b)=\mathcal{D}(a) b+a \mathcal{D}(b), \quad a, b \in \mathfrak{A} .
$$

A linear mapping $\mathcal{L}: \mathfrak{A} \rightarrow \mathfrak{X}$ is called a Lie derivation if

$$
\mathcal{L}[a, b]=[\mathcal{L}(a), b]+[a, \mathcal{L}(b)], \quad a, b \in \mathfrak{A},
$$

where $[\cdot, \cdot]$ stands for the Lie bracket. Trivially every derivation is a Lie derivation. If $\mathcal{D}: \mathfrak{A} \rightarrow \mathfrak{A}$ is a derivation and $\ell: \mathfrak{A} \rightarrow Z(\mathfrak{A})(:=$ the center of $\mathfrak{A})$ is a linear map, then $\mathcal{D}+\ell$ is a Lie derivation if and only if $\ell([a, b])=0$, for all

Received: 24.04.2016. Accepted: 16.09.2016. Published online: 18.12.2016.

(2010) Mathematics Subject Classification: 16W25, 15A78, 47B47.

Key words and phrases: derivation, Lie derivation, trivial extension algebra, triangular algebra. 
$a, b \in \mathfrak{A}$. Lie derivations of this form are called proper Lie derivations. A problem that we are dealing with is studying those conditions on an algebra such that every Lie derivation on it is proper. We say that an algebra $\mathfrak{A}$ has Lie derivation property if every Lie derivation on $\mathfrak{A}$ is proper.

Martindale [10] was the first one who showed that every Lie derivation on certain primitive ring is proper. Cheung [3] initiated the study of various mappings on triangular algebras; in particular, he investigated the properness of Lie derivations on triangular algebras (see also [4, 9, 12]). Cheung's results [4] have recently extended by Du and Wang [5] for a generalized matrix algebras. Wang [14] studied Lie $n$-derivations on a unital algebra with a nontrivial idempotent. Lie triple derivations on a unital algebra with a nontrivial idempotent have recently investigated by Benkovič [2].

In this paper we study Lie derivations on a trivial extension algebra. Let $\mathfrak{X}$ be an $\mathfrak{A}$-bimodule, then the direct product $\mathfrak{A} \times \mathfrak{X}$ together with the pairwise addition, scalar product and the algebra multiplication defined by

$$
(a, x)(b, y)=(a b, a y+x b), \quad a, b \in \mathfrak{A}, x, y \in \mathfrak{X},
$$

is a unital algebra which is called a trivial extension of $\mathfrak{A}$ by $\mathfrak{X}$ and will be denoted by $\mathfrak{A} \ltimes \mathfrak{X}$. For example, every triangular algebra $\operatorname{Tr}(\mathcal{A}, \mathfrak{X}, \mathcal{B})$ is a trivial extension algebra. Indeed, it can be identified with the trivial extension algebra $(\mathcal{A} \oplus \mathcal{B}) \ltimes \mathfrak{X} ;$ (see Sec. 3).

Trivial extension algebras are known as a rich source of (counter-)examples in various situations in functional analysis. Some aspects of (Banach) algebras of this type have been investigated in [1 and [15]. Derivations into various duals of a trivial extension (Banach) algebra studied in [15]. Jordan (higher) derivations on a trivial extension algebra are discussed in [11] (see also [6], 8] and [7]).

The main aim of this paper is providing some conditions under which a trivial extension algebra has the Lie derivation property. We are mainly dealing with those $\mathfrak{A} \ltimes \mathfrak{X}$ for which $\mathfrak{A}$ enjoys a nontrivial idempotent $p$ satisfying

$$
p x q=x,
$$

for all $x \in \mathfrak{X}$, where $q=1-p$. A triangular algebra is the main example of a trivial extension algebra satisfying $(\star)$.

In Section 2, we characterize the properness of a Lie derivation on $\mathfrak{A} \ltimes \mathfrak{X}$ (Theorem 2.2, from which we derive Theorem 2.3, providing some sufficient conditions ensuring the Lie derivation property for $\mathfrak{A} \ltimes \mathfrak{X}$. In Section 3 , we apply our results for a triangular algebra, recovering some results of [4]. 


\section{Proper Lie derivations on $\mathfrak{A} \ltimes \mathfrak{X}$}

We commence with the following elementary lemma describing the structures of derivations and Lie derivations on a trivial extension algebra $\mathfrak{A} \ltimes \mathfrak{X}$.

Lemma 2.1. Let $\mathfrak{A}$ be a unital algebra and $\mathfrak{X}$ be an $\mathfrak{A}$-bimodule. Then every linear map $\mathcal{L}: \mathfrak{A} \ltimes \mathfrak{X} \rightarrow \mathfrak{A} \ltimes \mathfrak{X}$ has the presentation

$$
\mathcal{L}(a, x)=\left(\mathcal{L}_{\mathfrak{A}}(a)+T(x), \mathcal{L}_{\mathfrak{X}}(a)+S(x)\right), \quad a \in \mathfrak{A}, x \in \mathfrak{X},
$$

for some linear mappings $\mathcal{L}_{\mathfrak{A}}: \mathfrak{A} \rightarrow \mathfrak{A}, \mathcal{L}_{\mathfrak{X}}: \mathfrak{A} \rightarrow \mathfrak{X}, T: \mathfrak{X} \rightarrow \mathfrak{A}$ and $S: \mathfrak{X} \rightarrow \mathfrak{X}$. Moreover,

- $\mathcal{L}$ is a Lie derivation if and only if

(a) $\mathcal{L}_{\mathfrak{A}}$ and $\mathcal{L}_{\mathfrak{X}}$ are Lie derivations;

(b) $T([a, x])=[a, T(x)]$ and $[T(x), y]=[T(y), x]$;

(c) $S([a, x])=\left[\mathcal{L}_{\mathfrak{A}}(a), x\right]+[a, S(x)]$,

for all $a \in \mathfrak{A}, x, y \in \mathfrak{X}$.

- $\mathcal{L}$ is a derivation if and only if

(i) $\mathcal{L}_{\mathfrak{A}}$ and $\mathcal{L}_{\mathfrak{X}}$ are derivations;

(ii) $T(a x)=a T(x), T(x a)=T(x) a$ and $x T(y)+T(x) y=0$;

(iii) $S(a x)=a S(x)+\mathcal{L}_{\mathfrak{A}}(a) x$ and $S(x a)=S(x) a+x \mathcal{L}_{\mathfrak{A}}(a)$, for all $a \in \mathfrak{A}, x, y \in \mathfrak{X}$.

It can be simply verified that the center $Z(\mathfrak{A} \ltimes \mathfrak{X})$ of $\mathfrak{A} \ltimes \mathfrak{X}$ is

$$
\begin{aligned}
Z(\mathfrak{A} \ltimes \mathfrak{X}) & =\{(a, x) ; a \in Z(\mathfrak{A}),[b, x]=0=[a, y] \text { for all } b \in \mathfrak{A}, y \in \mathfrak{X}\} \\
& =\pi_{\mathfrak{A}}(Z(\mathfrak{A} \ltimes \mathfrak{X})) \times \pi_{\mathfrak{X}}(Z(\mathfrak{A} \ltimes \mathfrak{X})),
\end{aligned}
$$

where $\pi_{\mathfrak{A}}: \mathfrak{A} \ltimes \mathfrak{X} \rightarrow \mathfrak{A}$ and $\pi_{\mathfrak{X}}: \mathfrak{A} \ltimes \mathfrak{X} \rightarrow \mathfrak{X}$ are the natural projections given by $\pi_{\mathfrak{A}}(a, x)=a$ and $\pi_{\mathfrak{X}}(a, x)=x$, respectively.

It should be noticed that, if $\mathfrak{A} \ltimes \mathfrak{X}$ satisfies $(\star)$, then the equality $[p, x]=0$ implies $x=0$, for any $x \in \mathfrak{X}$. This leads to $\pi_{\mathfrak{X}}(Z(\mathfrak{A} \ltimes \mathfrak{X}))=\{0\}$, and so

$$
\begin{aligned}
Z(\mathfrak{A} \ltimes \mathfrak{X}) & =\{(a, 0) ; a \in Z(\mathfrak{A}),[a, x]=0 \text { for all } x \in \mathfrak{X}\} \\
& =\pi_{\mathfrak{A}}(Z(\mathfrak{A} \ltimes \mathfrak{X})) \times\{0\} .
\end{aligned}
$$

Further, the property $(\star)$ also implies the following simplifications on the module operations which will be frequently used in the sequel

$$
q x=0=x p, p x=x=x q, p a p x=a x, x q a q=x a, \quad a \in \mathfrak{A}, x \in \mathfrak{X}
$$


The following characterization theorem which is a generalization of 4 , Theorem 6] studies the properness of a Lie derivation on $\mathfrak{A} \ltimes \mathfrak{X}$. Before proceeding, we recall that an $\mathfrak{A}$-bimodule $\mathfrak{X}$ is called 2-torsion free if $2 x=0$ implies $x=0$, for any $x \in \mathfrak{X}$.

Theorem 2.2. Suppose that the trivial extension algebra $\mathfrak{A} \ltimes \mathfrak{X}$ satisfies $(\star)$ and that both $\mathfrak{A}$ and $\mathfrak{X}$ are 2 -torsion free. Then a Lie derivation $\mathcal{L}$ on $\mathfrak{A} \ltimes \mathfrak{X}$ of the form

$$
\mathcal{L}(a, x)=\left(\mathcal{L}_{\mathfrak{A}}(a)+T(x), \mathcal{L}_{\mathfrak{X}}(a)+S(x)\right), \quad a \in \mathfrak{A}, x \in \mathfrak{X},
$$

is proper if and only if there exists a linear map $\ell_{\mathfrak{A}}: \mathfrak{A} \rightarrow Z(\mathfrak{A})$ satisfying the following conditions:

(i) $\mathcal{L}_{\mathfrak{A}}-\ell_{\mathfrak{A}}$ is a derivation on $\mathfrak{A}$.

(ii) $\left[\ell_{\mathfrak{A}}(\right.$ pap $\left.), x\right]=0=\left[\ell_{\mathfrak{A}}(q a q), x\right]$ for all $a \in \mathfrak{A}, x \in \mathfrak{X}$.

Proof. By Lemma 2.1 every Lie derivation on $\mathfrak{A} \ltimes \mathfrak{X}$ can be expressed in the from

$$
\mathcal{L}(a, x)=\left(\mathcal{L}_{\mathfrak{A}}(a)+T(x), \mathcal{L}_{\mathfrak{X}}(a)+S(x)\right),
$$

where $\mathcal{L}_{\mathfrak{A}}: \mathfrak{A} \rightarrow \mathfrak{A}, \mathcal{L}_{\mathfrak{X}}: \mathfrak{A} \rightarrow \mathfrak{X}$ are Lie derivations and $T: \mathfrak{X} \rightarrow \mathfrak{A}, S: \mathfrak{X} \rightarrow \mathfrak{X}$ are linear mappings satisfying

$$
\begin{aligned}
T([a, x])=[a, T(x)], \quad[T(x), y] & =[T(y), x], \\
& \text { and } S([a, x])=\left[\mathcal{L}_{\mathfrak{A}}(a), x\right]+[a, S(x)],
\end{aligned}
$$

for all $a \in \mathfrak{A}, x, y \in \mathfrak{X}$.

To prove "if" part, we set

$$
\begin{aligned}
& \mathcal{D}(a, x)=\left(\left(\mathcal{L}_{\mathfrak{A}}-\ell_{\mathfrak{A}}\right)(a)+T(x), \mathcal{L}_{\mathfrak{X}}(a)+S(x)\right) \\
& \quad \text { and } \quad \ell(a, x)=\left(\ell_{\mathfrak{A}}(a), 0\right), \quad a \in \mathfrak{A}, x \in \mathfrak{X} .
\end{aligned}
$$

Then clearly $\mathcal{L}=\mathcal{D}+\ell$. That $\ell$ is linear and $\ell(\mathfrak{A} \ltimes \mathfrak{X}) \subseteq Z(\mathfrak{A} \ltimes \mathfrak{X})$ follows trivially from $\ell_{\mathfrak{A}}(\mathfrak{A}) \subseteq Z(\mathfrak{A})$ and 2.2 . It remains to show that $\mathcal{D}$ is a derivation on $\mathfrak{A} \ltimes \mathfrak{X}$. To do this we use Lemma 2.1. It should be mentioned that in the rest of proof we frequently making use the equalities in $(2.3)$. First we have,

$$
\begin{aligned}
S(a x) & =S([\text { pap }, x]) \\
& =\left[\mathcal{L}_{\mathfrak{A}}(\text { pap }), x\right]+[\operatorname{pap}, S(x)]
\end{aligned}
$$




$$
\begin{aligned}
= & {\left[\left(\mathcal{L}_{\mathfrak{A}}-\ell_{\mathfrak{A}}\right)(\text { pap }), x\right]+\left[\ell_{\mathfrak{A}}(\text { pap }), x\right]+a S(x) } \\
= & {\left[\left(\left(\mathcal{L}_{\mathfrak{A}}-\ell_{\mathfrak{A}}\right)(p) a p+p\left(\mathcal{L}_{\mathfrak{A}}-\ell_{\mathfrak{A}}\right)(a) p+p a\left(\mathcal{L}_{\mathfrak{A}}-\ell_{\mathfrak{A}}\right)(p)\right), x\right] } \\
& +\left[\ell_{\mathfrak{A}}(\text { pap }), x\right]+a S(x) \\
= & \left(\mathcal{L}_{\mathfrak{A}}-\ell_{\mathfrak{A}}\right)(a) x+\left[\ell_{\mathfrak{A}}(\text { pap }), x\right]+a S(x),
\end{aligned}
$$

for all $a \in \mathfrak{A}, x \in \mathfrak{X}$. Now the condition $\left[\ell_{\mathfrak{A}}(\right.$ pap $\left.), x\right]=0$ implies that $S(x a)=$ $\left(\mathcal{L}_{\mathfrak{A}}-\ell_{\mathfrak{A}}\right)(a) x+a S(x)$. With a similar procedure as above, from $\left[\ell_{\mathfrak{A}}(q a q), x\right]=$ 0 we get $S(x a)=x\left(\mathcal{L}_{\mathfrak{A}}-\ell_{\mathfrak{A}}\right)(a)+S(x) a$ for all $a \in \mathfrak{A}, x \in \mathfrak{X}$.

From the equality

$$
T(x)=T([p, x])=[p, T(x)]=p T(x)-T(x) p, \quad x \in \mathfrak{X},
$$

we arrive at $y T(x)=0=T(x) y$ and so $y T(x)+T(y) x=0$ for all $y, x \in \mathfrak{X}$. It also follows that $p T(x) p=0, q T(x) q=0$ and $q T(x) p=0$ for all $x \in \mathfrak{X}$; note that $\mathfrak{A}$ is 2 -torsion free.

The equality

$$
0=T([q a p, x])=[q a p, T(x)]=q a p T(x)-T(x) q a p, \quad a \in \mathfrak{A}, x \in \mathfrak{X},
$$

gives $q a p T(x)=T(x) q a p$ for all $a \in \mathfrak{A}, x \in \mathfrak{X}$. The latter relation together with the equality

$$
T(a x)=T[p a, x]=p a T(x)-T(x) p a, \quad a \in \mathfrak{A}, x \in \mathfrak{X},
$$

lead us to $T(a x)=p T(a x) q=p a T(x) q=a T(x)$ for all $a \in \mathfrak{A}, x \in \mathfrak{X}$. By a similar argument we get $T(x a)=T(x) a$ for all $a \in \mathfrak{A}, x \in \mathfrak{X}$.

Next, we set $\phi(a)=\mathcal{L}_{\mathfrak{X}}(p a q)$, then $\phi$ is a derivation. Indeed, for each $a, b \in \mathfrak{A}$,

$$
\begin{aligned}
\phi(a b)= & \mathcal{L}_{\mathfrak{X}}(p a b q)=\mathcal{L}_{\mathfrak{X}}([p a, p b q])+\mathcal{L}_{\mathfrak{X}}([p a q, b q]) \\
= & \mathcal{L}_{\mathfrak{X}}(p a) p b q-p b q \mathcal{L}_{\mathfrak{X}}(p a)+p a \mathcal{L}_{\mathfrak{X}}(p b q)-\mathcal{L}_{\mathfrak{X}}(p b q) p a \\
& +\mathcal{L}_{\mathfrak{X}}(p a q) b q-b q \mathcal{L}_{\mathfrak{X}}(p a q)+p a q \mathcal{L}_{\mathfrak{X}}(b q)-\mathcal{L}_{\mathfrak{X}}(b q) p a q \\
= & a \phi(b)+\phi(a) b .
\end{aligned}
$$

As $\mathfrak{X}$ is 2 -torsion free, the identity

$$
\mathcal{L}_{\mathfrak{X}}(q a p)=\mathcal{L}_{\mathfrak{X}}([q a p, p])=\left[\mathcal{L}_{\mathfrak{X}}(q a p), p\right]+\left[q a p, \mathcal{L}_{\mathfrak{X}}(p)\right]=-\mathcal{L}_{\mathfrak{X}}(q a p),
$$

implies that $\mathcal{L}_{\mathfrak{X}}(q a p)=0$ for all $a \in \mathfrak{A}$. 
As $\mathcal{L}_{\mathfrak{X}}([p a p, q a q])=0$, for all $a \in \mathfrak{A}$, we get,

$$
\mathcal{L}_{\mathfrak{X}}(\text { pap }) q a q=-\operatorname{pap} \mathcal{L}_{\mathfrak{X}}(q a q) .
$$

Substituting $a$ with $q a q+p$ (resp. pap $+q)$ in $(2.5)$, leads to $p \mathcal{L}_{\mathfrak{X}}(q a q) q=$ $-\mathcal{L}_{\mathfrak{X}}(p) a$ (resp. $\left.p \mathcal{L}_{\mathfrak{X}}(p a p) q=a \mathcal{L}_{\mathfrak{X}}(p)\right)$, for all $a \in \mathfrak{A}$. We use the latter relations to prove that $\mathcal{L}_{\mathfrak{X}}$ is the sum of an inner derivation (implemented by $\mathcal{L}_{\mathfrak{X}}(p)$ ) and $\phi$, and so it is a derivation. Indeed, for each $a \in \mathfrak{A}$,

$$
\begin{aligned}
\mathcal{L}_{\mathfrak{X}}(a) & =\mathcal{L}_{\mathfrak{X}}(\text { pap })+\mathcal{L}_{\mathfrak{X}}(q a q)+\mathcal{L}_{\mathfrak{X}}(\text { paq }) \\
& =p \mathcal{L}_{\mathfrak{X}}(p a p) q+p \mathcal{L}_{\mathfrak{X}}(q a q) q+\phi(a) \\
& =a \mathcal{L}_{\mathfrak{X}}(p)-\mathcal{L}_{\mathfrak{X}}(p) a+\phi(a) .
\end{aligned}
$$

Now Lemma 2.1 confirms that $\mathcal{D}$ is a derivation on $\mathfrak{A} \ltimes \mathfrak{X}$, and so $\mathcal{L}$ is proper, as claimed.

For the converse, suppose that $\mathcal{L}$ is proper, that is, $\mathcal{L}=\mathcal{D}+\ell$, where $\mathcal{D}$ is a derivation and $\ell$ is a center valued linear map on $\mathfrak{A} \ltimes \mathfrak{X}$. Then, from (2.2), we get $\ell(\mathfrak{A} \ltimes \mathfrak{X}) \subseteq \pi_{\mathfrak{A}}(Z(\mathfrak{A} \ltimes \mathfrak{X})) \times\{0\}$, and this implies that $\ell$ has the presentation $\ell(a, x)=\left(\ell_{\mathfrak{A}}(a), 0\right)$ with $\left[\ell_{A}(a), x\right]=0$, for all $a \in \mathfrak{A}, x \in \mathfrak{X}$, for some linear map $\ell_{\mathfrak{A}}: \mathfrak{A} \rightarrow Z(\mathfrak{A})$. On the other hand, $\mathcal{L}-\ell=\mathcal{D}$ is a derivation on $\mathfrak{A} \ltimes \mathfrak{X}$ and so, by Lemma 2.1, $\mathcal{L}_{\mathfrak{A}}-\ell_{\mathfrak{A}}$ is a derivation on $\mathfrak{A}$, as required.

Applying Theorem 2.2, we come to the next main result providing some sufficient conditions ensuring the Lie derivation property for $\mathfrak{A} \ltimes \mathfrak{X}$. Note that we use the projection maps $\pi_{p \mathfrak{A} p}: \mathfrak{A} \ltimes \mathfrak{X} \rightarrow \mathfrak{A}$ and $\pi_{q \mathfrak{A} q}: \mathfrak{A} \ltimes \mathfrak{X} \rightarrow \mathfrak{A}$ defined by $\pi_{p \mathfrak{A} p}(a, x)=p a p$ and $\pi_{q \mathfrak{A} q}(a, x)=q a q$, respectively. Before proceeding, we also introduce an auxiliary subalgebra $\mathcal{W}_{\mathfrak{A}}$ associated to $\mathfrak{A}$. For an algebra $\mathfrak{A}$, we denote by $\mathcal{W}_{\mathfrak{A}}$ the smallest subalgebra of $\mathfrak{A}$ contains all commutators and idempotents. We are especially dealing with those algebras satisfying $\mathcal{W}_{\mathfrak{A}}=\mathfrak{A}$. Some known examples of algebras satisfying $\mathcal{W}_{\mathfrak{A}}=\mathfrak{A}$ are: the full matrix algebra $\mathfrak{A}=M_{n}(A), n \geqslant 2$, where $A$ is a unital algebra, and also every simple unital algebra $\mathfrak{A}$ with a nontrivial idempotent.

TheOREM 2.3. Suppose that the trivial extension algebra $\mathfrak{A} \ltimes \mathfrak{X}$ satisfies $(\star)$ and that both $\mathfrak{A}$ and $\mathfrak{X}$ are 2 -torsion free. Then $\mathfrak{A} \ltimes \mathfrak{X}$ has Lie derivation property if the following two conditions are satisfied:

(I) $\mathfrak{A}$ has Lie derivation property.

(II) One of the following three conditions hold:

(i) $\mathcal{W}_{p \mathfrak{A} p}=p \mathfrak{A} p$ and $\mathcal{W}_{q \mathfrak{A} q}=q \mathfrak{A} q$. 
(ii) $Z(p \mathfrak{A} p)=\pi_{p \mathfrak{A} p}(Z(\mathfrak{A} \ltimes \mathfrak{X}))$ and $p \mathfrak{A} q$ is faithful as a right $q \mathfrak{A} q$-module.

(iii) $Z(q \mathfrak{A} q)=\pi_{q \mathfrak{A} q}(Z(\mathfrak{A} \ltimes \mathfrak{X}))$ and $p \mathfrak{A} q$ is faithful as a left $p \mathfrak{A} p$-module.

Proof. Let $\mathcal{L}$ be a Lie derivation on $\mathfrak{A} \ltimes \mathfrak{X}$ with the presentation as given in Lemma 2.1. Since $\mathcal{L}_{\mathfrak{A}}$ is a Lie derivation and (by (I)) $\mathfrak{A}$ has Lie derivation property, there exists a linear map $\ell_{\mathfrak{A}}: \mathfrak{A} \rightarrow Z(\mathfrak{A})$ such that $\mathcal{L}_{\mathfrak{A}}-\ell_{\mathfrak{A}}$ is a derivation on $\mathfrak{A}$ (and so $\ell_{\mathfrak{A}}$ vanishes on commutators of $\mathfrak{A}$ ). It is enough to show that, under either conditions of (II), $\ell_{\mathfrak{A}}$ satisfies Theorem 2.2(ii); that is, $\left[\ell_{\mathfrak{A}}(\right.$ pap $\left.), x\right]=0=\left[\ell_{\mathfrak{A}}(q a q), x\right]$ for all $a \in \mathfrak{A}, x \in \mathfrak{X}$.

To prove the conclusion, we consider the subset $\mathfrak{A}^{\prime}=\left\{\right.$ pap $:\left[\ell_{\mathfrak{A}}(\right.$ pap $\left.), x\right]=$ 0 , for all $x \in \mathfrak{X}\}$ of $p \mathfrak{A} p$. We are going to show that $\mathfrak{A}^{\prime}$ is a subalgebra of $p \mathfrak{A} p$ including all idempotents and commutators of $p \mathfrak{A} p$. First, we shall prove that $\mathfrak{A}^{\prime}$ is a subalgebra. That $\mathfrak{A}^{\prime}$ is an $\mathbf{R}$-submodule of $\mathfrak{A}$ follows from the linearity of $\ell_{\mathfrak{A}}$. The following identity confirms that $\mathfrak{A}^{\prime}$ is closed under multiplication

$$
\left[\ell_{\mathfrak{A}}(\text { papbp) }, x]=\left[\ell_{\mathfrak{A}}(\text { pap }), b x\right]+\left[\ell_{\mathfrak{A}}(p b p), a x\right], \quad a, b \in \mathfrak{A}, x \in \mathfrak{X} .\right.
$$

To prove (2.6), note that from the identity (2.4) we have

$$
S(a x)=\left(\mathcal{L}_{\mathfrak{A}}-\ell_{\mathfrak{A}}\right)(a) x+\left[\ell_{\mathfrak{A}}(\text { pap }), x\right]+a S(x), \quad a \in \mathfrak{A}, x \in \mathfrak{X} .
$$

Applying 2.7) for $a b$ we have,

$$
S(a b x)=\left(\mathcal{L}_{\mathfrak{A}}-\ell_{\mathfrak{A}}\right)(a b) x+\left[\ell_{\mathfrak{A}}(p a b p), x\right]+a b S(x) .
$$

On the other hand, since $a\left[\ell_{\mathfrak{A}}(p b p), x\right]=\left[\ell_{\mathfrak{A}}(p b p), a x\right]$, we have,

$$
\begin{aligned}
S(a b x)= & \left(\mathcal{L}_{\mathfrak{A}}-\ell_{\mathfrak{A}}\right)(a) b x+\left[\ell_{\mathfrak{A}}(\text { pap }), b x\right]+a S(b x) \\
= & \left(\mathcal{L}_{\mathfrak{A}}-\ell_{\mathfrak{A}}\right)(a) b x+\left[\ell_{\mathfrak{A}}(\text { pap }), b x\right]+a\left(\mathcal{L}_{\mathfrak{A}}-\ell_{\mathfrak{A}}\right)(b) x \\
& +\left[\ell_{\mathfrak{A}}(p b p), a x\right]+a b S(x) .
\end{aligned}
$$

Using the fact that $\mathcal{L}_{\mathfrak{A}}-\ell_{\mathfrak{A}}$ is a derivation, then a comparison of the latter equation and (2.8) leads to

$$
\left[\ell_{\mathfrak{A}}(p a b p), x\right]=\left[\ell_{\mathfrak{A}}(p a p), b x\right]+\left[\ell_{\mathfrak{A}}(p b p), a x\right]
$$

for all $a, b \in \mathfrak{A}, x \in \mathfrak{X}$, which trivially implies 2.6.

Next, we claim that $\mathfrak{A}^{\prime}$ contains all idempotents of $p \mathfrak{A} p$. First note that, if one puts $a=b$ in (2.6), then

$$
\left[\ell_{\mathfrak{A}}\left((\text { pap })^{2}\right), x\right]=\left[\ell_{\mathfrak{A}}(\text { pap }), 2 a x\right], \quad a \in \mathfrak{A}, x \in \mathfrak{X} .
$$


This follows that

$$
\begin{aligned}
{\left[\ell_{\mathfrak{A}}\left((\text { pap })^{3}\right), x\right] } & =\left[\ell_{\mathfrak{A}}\left((\text { pap })^{2}(\text { pap })\right), x\right] \\
& =\left[\ell_{\mathfrak{A}}(\text { pap }), 3 a^{2} x\right], \quad a \in \mathfrak{A}, x \in \mathfrak{X}
\end{aligned}
$$

Suppose that $p a p \in p \mathfrak{A} p$ is an idempotent, that is, $(\text { pap })^{2}=p a p$. By 2.9 and 2.10 , we arrive at

$$
\begin{aligned}
{\left[\ell_{\mathfrak{A}}(\text { pap }), x\right] } & =\left[\ell_{\mathfrak{A}}\left(3(\text { pap })^{2}-2(\text { pap })^{3}\right), x\right] \\
& =3\left[\ell_{\mathfrak{A}}(\text { pap }), 2 a x\right]-2\left[\ell_{\mathfrak{A}}(\text { pap }), 3 a^{2} x\right] \\
& =\left[\ell_{\mathfrak{A}}(\text { pap }),\left(6(\text { pap })-6(\text { pap })^{2}\right) x\right]=0
\end{aligned}
$$

and this says that the idempotent pap lies in $\mathfrak{A}^{\prime}$.

Further, that $\mathfrak{A}^{\prime}$ contains all commutatorts follows trivially from the fact that $\ell_{\mathfrak{A}}$ vanishes on commutators. We thus have proved that $\mathfrak{A}^{\prime}$ is a subalgebra of $p \mathfrak{A} p$ contains all idempotents and commutators. Now the assumption $\mathcal{W}_{p \mathfrak{A} p}=p \mathfrak{A} p$ in (i) gives $\mathfrak{A}^{\prime}=p \mathfrak{A} p$, that is, $\left[\ell_{\mathfrak{A}}(p a p), x\right]=0$ for every $a \in \mathfrak{A}, x \in \mathfrak{X}$. A similar argument shows that, if $\mathcal{W}_{q \mathfrak{A} q}=q \mathfrak{A} q$, then $\left[\ell_{\mathfrak{A}}(q a q), x\right]=0$ for every $a \in \mathfrak{A}, x \in \mathfrak{X}$.

Now suppose that $Z(p \mathfrak{A} p)=\pi_{p \mathfrak{A} p}(Z(\mathfrak{A} \ltimes \mathfrak{X}))$ and $p \mathfrak{A} q$ is faithful as a right $q \mathfrak{A} q$-module. In this case, to prove $\left[\ell_{\mathfrak{A}}(\right.$ pap $\left.), x\right]=0=\left[\ell_{\mathfrak{A}}(q a q), x\right]$ for all $a \in \mathfrak{A}, x \in \mathfrak{X}$, we actually shall show that $[Z(\mathfrak{A}), \mathfrak{X}]=0$. To this end, as the algebra $\mathfrak{A}$ enjoys the Peirce decomposition $\mathfrak{A}=p \mathfrak{A} p+p \mathfrak{A} q+q \mathfrak{A} p+q \mathfrak{A} q$, a direct verification reveals that

$$
\begin{aligned}
Z(\mathfrak{A})= & \{a \in \mathfrak{A} ; \text { pap } \in Z(p \mathfrak{A} p), q a q \in Z(q \mathfrak{A} q), \\
& \text { papm }=\text { mqaq, npap = qaqn for all } m \in p \mathfrak{A} q, n \in q \mathfrak{A} p\} .
\end{aligned}
$$

Combining the latter equality to that in 2.2 we arrive at

$$
\begin{aligned}
Z(\mathfrak{A} \ltimes \mathfrak{X})= & \{(a, 0) ; a \in \mathfrak{A}, p a p \in Z(p \mathfrak{A} p), q a q \in Z(q \mathfrak{A} q), \text { papm }=\text { mqaq, } \\
& n p a p=q a q n,[a, x]=0 \text { for all } m \in p \mathfrak{A} q, n \in q \mathfrak{A} p, x \in \mathfrak{X}\} .
\end{aligned}
$$

Let $a \in Z(\mathfrak{A})$. Since $p a p \in Z(p \mathfrak{A} p)$ and $Z(p \mathfrak{A} p)=\pi_{p \mathfrak{A} p}(Z(\mathfrak{A} \ltimes \mathfrak{X}))$, there exists an element $\left(a^{\prime}, 0\right) \in Z(\mathfrak{A} \ltimes \mathfrak{X})$ such that $p a p=\pi_{p \mathfrak{A} p}\left(a^{\prime}, 0\right)=p a^{\prime} p$. It follows that $m q a q=p a p m=p a^{\prime} p m=m q a^{\prime} q$ for each $m \in p \mathfrak{A} q$. Since $p \mathfrak{A} q$ is a faithful right $q \mathfrak{A} q$-module, we get $q a q=q a^{\prime} q$, and so $a=p a p+q a q=p a^{\prime} p+q a^{\prime} q=a^{\prime}$. In particular, $(a, 0) \in Z(\mathfrak{A} \ltimes \mathfrak{X})$ and so $[a, x]=0$ for all $x \in \mathfrak{X}$, as claimed. 
Similarly, if $Z(q \mathfrak{A} q)=\pi_{q \mathfrak{A} q}(Z(\mathfrak{A} \ltimes \mathfrak{X}))$ and $p \mathfrak{A} q$ is faithful as a left $p \mathfrak{A} p$ module, then the equality $[Z(\mathfrak{A}), \mathfrak{X}]=0$ holds, which completes the proof.

As the following example demonstrates, the Lie derivation property of $\mathfrak{A}$ in Theorem 2.3 is essential.

EXAMPLE 2.4. Let $\mathfrak{A}$ be a unital algebra with a nontrivial idempotent $p$, which does not have Lie derivation property. Let $\mathcal{L}_{\mathfrak{A}}$ be a non-proper Lie derivation on $\mathfrak{A}$ and $\mathfrak{X}$ be an $\mathfrak{A}$-bimodule such that $p x q=x$ and $\left[\mathcal{L}_{\mathfrak{A}}(a), x\right]=0$, for all $a \in \mathfrak{A}, x \in \mathfrak{X}$. Then a direct verification shows that $\mathcal{L}(a, x)=\left(\mathcal{L}_{\mathfrak{A}}(a), 0\right)$, is actually a non-proper Lie derivation on $\mathfrak{A} \ltimes \mathfrak{X}$.

To see a concrete example of a pair $\mathfrak{A}, \mathfrak{X}$ satisfying the aforementioned conditions, let $\mathfrak{A}$ be the triangular matrix algebra as given in [4. Example 8] and let $\mathfrak{X}=\mathbb{R}$ equipped with the module operations: $x \cdot\left(a_{i j}\right)=x a_{11}$, $\left(a_{i j}\right) \cdot x=a_{44} x,\left(\left(a_{i j}\right) \in \mathfrak{A}\right.$ and $\left.x \in \mathbb{R}\right)$.

The above example and Theorem 2.3 confirm that, the Lie derivation property of $\mathfrak{A}$ plays a key role for the Lie derivation property of $\mathfrak{A} \ltimes \mathfrak{X}$. In this respect, Lie derivation property of a unital algebra containing a nontrivial idempotent has already studied by Benkovič [2, Theorem 5.3] (see also the case $n=2$ of a result given by Wang [14, Theorem 2.1]). About the Lie derivation property of a unital algebra with a nontrivial idempotent, we quote the following result from the first and third authors [13], which extended the aforementioned results.

Proposition 2.5 ([13, Corollary 4.3]). Let $\mathfrak{A}$ be a 2-torsion free unital algebra with a nontrivial idempotent $p$ and $q=1-p$. Then $\mathfrak{A}$ has Lie derivation property if the following three conditions hold:

(I) $Z(q \mathfrak{A} q)=Z(\mathfrak{A}) q$ and $p \mathfrak{A} q$ is a faithful left $p \mathfrak{A} p$-module; or $\mathcal{W}_{p \mathfrak{A} p}=p \mathfrak{A} p$ and $p \mathfrak{A} q$ is a faithful left $p \mathfrak{A} p$-module; or $p \mathfrak{A} p$ has Lie derivation property and $\mathcal{W}_{p \mathfrak{A l} p}=p \mathfrak{A} p$.

(II) $Z(p \mathfrak{A} p)=Z(\mathfrak{A}) p$ and $q \mathfrak{A} p$ is a faithful right $q \mathfrak{A} q$-module; or $\mathcal{W}_{q \mathfrak{A} q}=q \mathfrak{A} q$ and $q \mathfrak{A} p$ is a faithful right $q \mathfrak{A} q$-module; or $q \mathfrak{A} q$ has Lie derivation property and $\mathcal{W}_{q \mathfrak{A} q}=q \mathfrak{A} q$

(III) One of the following assertions holds:

(i) Either $p \mathfrak{A} p$ or $q \mathfrak{A} q$ does not contain nonzero central ideals.

(ii) $p \mathfrak{A} p$ and $q \mathfrak{A} q$ are domain.

(iii) Either $p \mathfrak{A} q$ or $q \mathfrak{A} p$ is strongly faithful.

It should also be remarked that if $p \mathfrak{A} q \mathfrak{A} p=0$ and $q \mathfrak{A} p \mathfrak{A} q=0$, then the condition (III) in the above proposition is superfluous and can be dropped from the hypotheses, (see also [2, Remark 5.4]). One may apply Proposition 2.5 to show that, the algebra $\mathfrak{A}=B(X)$, of all bounded operators on a 
Banach space $X$ with dimension greater than 2, as well as, the full matrix algebra $\mathfrak{A}=M_{n}(A), n \geqslant 2$, where $A$ is a 2-torsion free unital algebra, have the Lie derivation property.

Illustrating Theorem 2.3 and Proposition 2.5, in the following we give an example of a trivial extension algebra, which is not a triangular algebra, having Lie derivation property .

EXAmple 2.6. We consider the next subalgebra $\mathfrak{A}$ of $M_{4}(\mathbb{R})$ with a nontrivial idempotent $p$ as follows;

$$
\mathfrak{A}=\left\{\left(\begin{array}{cccc}
a & 0 & 0 & 0 \\
0 & b & u & 0 \\
0 & 0 & c & 0 \\
0 & 0 & 0 & d
\end{array}\right) \mid a, b, c, d, u \in \mathbb{R}\right\}, \quad p=\left(\begin{array}{llll}
0 & 0 & 0 & 0 \\
0 & 0 & 0 & 0 \\
0 & 0 & 1 & 0 \\
0 & 0 & 0 & 0
\end{array}\right) .
$$

One can directly check that $p \mathfrak{A} p \cong \mathbb{R}$ and $q \mathfrak{A} q \cong \mathbb{R}^{3}$ (where the algebras $\mathbb{R}$ and $\mathbb{R}^{3}$ are equipped with their natural pointwise multiplications). In particular, $p \mathfrak{A} p, q \mathfrak{A} q$ have Lie derivation property, $\mathcal{W}_{p \mathfrak{A} p}=p \mathfrak{A} p, \mathcal{W}_{q \mathfrak{A} q}=q \mathfrak{A} q$ and $p \mathfrak{A} p$ does not contain nonzero central ideals. Thus, by virtue of Proposition 2.5, $\mathfrak{A}$ has Lie derivation property.

Further, $\mathfrak{X}=\mathbb{R}$ is an $\mathfrak{A}$-bimodule furnished with the module operations as

$$
\left(a_{i j}\right) \cdot x=a_{33} x, \quad x \cdot\left(a_{i j}\right)=x a_{22}, \quad\left(a_{i j}\right) \in \mathfrak{A}, x \in \mathbb{R} .
$$

Then clearly the trivial extension algebra $\mathfrak{A} \ltimes \mathbb{R}$ satisfies the condition $(\star)$; that is, $p x q=x$ for all $x \in \mathbb{R}$. So Theorem 2.3 guarantees that $\mathfrak{A} \ltimes \mathbb{R}$ has Lie derivation property. It is worthwhile mentioning that $\mathfrak{A} \ltimes \mathbb{R}$ is not a triangular algebra. This can be directly verified that, there is no nontrivial idempotent $P \in \mathfrak{A} \ltimes \mathbb{R}$ such that $P(\mathfrak{A} \ltimes \mathbb{R}) Q \neq 0$ and $Q(\mathfrak{A} \ltimes \mathbb{R}) P=0$, where $Q=1-P$ (see [3]).

\section{Application to triangular algebras}

We recall that a triangular algebra $\operatorname{Tri}(\mathcal{A}, \mathfrak{X}, \mathcal{B})$ is an algebra of the form

$$
\operatorname{Tri}(\mathcal{A}, \mathfrak{X}, \mathcal{B})=\left\{\left(\begin{array}{ll}
a & x \\
0 & b
\end{array}\right) \mid a \in \mathcal{A}, x \in \mathfrak{X}, b \in \mathcal{B}\right\},
$$

whose algebra operations are just like $2 \times 2$-matrix operations; where $\mathcal{A}$ and $\mathcal{B}$ are unital algebras and $\mathfrak{X}$ is an $(\mathcal{A}, \mathcal{B})$-bimodule; that is, a left $\mathcal{A}$-module 
and a right $\mathcal{B}$-module. One can easily check that $\operatorname{Tri}(\mathcal{A}, \mathfrak{X}, \mathcal{B})$ is isomorphic to the trivial extension algebra $(\mathcal{A} \oplus \mathcal{B}) \ltimes \mathfrak{X}$, where the algebra $\mathcal{A} \oplus \mathcal{B}$ has its usual pairwise operations and $\mathfrak{X}$ as an $(\mathcal{A} \oplus \mathcal{B})$-bimodule is equipped with the module operations

$$
(a \oplus b) x=a x \quad \text { and } \quad x(a \oplus b)=x b, \quad a \in \mathcal{A}, b \in \mathcal{B}, x \in \mathfrak{X} .
$$

Furthermore, the triangular algebra $\operatorname{Tri}(\mathcal{A}, \mathfrak{X}, \mathcal{B}) \cong(\mathcal{A} \oplus \mathcal{B}) \ltimes \mathfrak{X}$ satisfies the condition $(\star)$. Indeed, $p=\left(1_{\mathcal{A}}, 0\right)$ is a nontrivial idempotent, $q=\left(0,1_{\mathcal{B}}\right)$ and a direct verification shows that $p x q=x$, for all $x \in \mathfrak{X}$. Further, in this case for $\mathfrak{A}=\mathcal{A} \oplus \mathcal{B}$ we have,

$$
p \mathfrak{A} p \cong \mathcal{A}, \quad p \mathfrak{A} q=0, \quad q \mathfrak{A} p=0 \quad \text { and } \quad q \mathfrak{A} q \cong \mathcal{B} .
$$

It should be mentioned that in this case, for a Lie derivation $\mathcal{L}$ on $(\mathcal{A} \oplus$ $\mathcal{B}) \ltimes \mathfrak{X}$ with the presentation

$$
\mathcal{L}(a \oplus b, x)=\left(\mathcal{L}_{\mathcal{A} \oplus \mathcal{B}}(a \oplus b)+T(x), \mathcal{L}_{\mathfrak{X}}(a \oplus b)+S(x),\right) \quad(a \oplus b) \in \mathcal{A} \oplus \mathcal{B}, x \in \mathfrak{X},
$$

as given in Lemma 2.1. we conclude that $T=0$. Indeed, by Lemma 2.1(b), $T([a \oplus b, x])=[a \oplus b, T(x)]$ for all $a \in \mathcal{A}, b \in \mathcal{B}, x \in \mathfrak{X}$. Using the latter relation for $a=1, b=0$ implies that $T(x)=0$ for all $x \in \mathfrak{X}$.

A quick look at the proof of Theorem 2.2 reveals that, in this special case, as $T=0$ and $q \mathfrak{A} p=0$, we do not need the 2-torsion freeness of $\mathfrak{A}$ and $\mathfrak{X}$ in Theorems 2.2 and 2.3 .

A direct verification also reveals that, the direct sum $\mathfrak{A}=\mathcal{A} \oplus \mathcal{B}$ has Lie derivation property if and only if both $\mathcal{A}$ and $\mathcal{B}$ have Lie derivation property.

Now, by the above observations, as an immediate consequence of Theorem 2.3. we directly arrive at the following result of Cheung (see [4, Theorem 11]).

Corollary 3.1. Let $\mathcal{A}$ and $\mathcal{B}$ be unital algebras and let $\mathfrak{X}$ be an $(\mathcal{A}, \mathcal{B})$ bimodule. Then the triangular algebra $\mathcal{T}=\operatorname{Tri}(\mathcal{A}, \mathfrak{X}, \mathcal{B})$ has Lie derivation property if the following two conditions are satisfied:

(I) $\mathcal{A}$ and $\mathcal{B}$ have Lie derivation property.

(II) $\mathcal{W}_{\mathcal{A}}=\mathcal{A}$ and $\mathcal{W}_{\mathcal{B}}=\mathcal{B}$.

It should be remarked here that, in [4, Theorem 11], Cheung combined his hypotheses with some "faithfulness" conditions and the equalities $Z(\mathcal{A})=$ $\pi_{\mathcal{A}}(Z(\mathcal{T}))$ and/or $Z(\mathcal{B})=\pi_{B}(Z(\mathcal{T}))$. Combining the conditions " $\mathfrak{X}$ is faithful as a left $\mathcal{A}$-module" and " $\mathfrak{X}$ is faithful as a right $\mathcal{B}$-module" with those in the above corollary provides some more sufficient conditions ensuring the Lie derivation property for the triangular algebra $\operatorname{Tri}(\mathcal{A}, \mathfrak{X}, \mathcal{B})$. His results can be 
satisfactorily extended to a trivial extension algebra $\mathfrak{A} \ltimes \mathfrak{X}$ by employing the hypothesis " $\mathfrak{X}$ is loyal" instead of " $\mathfrak{X}$ is faithful".

We recall that, in the case where a unital algebra $\mathfrak{A}$ has a nontrivial idempotent $p$, an $\mathfrak{A}$-bimodule $\mathfrak{X}$ is said to be left loyal if $a \mathfrak{X}=0$ implies that pap $=0$, right loyal if $\mathfrak{X} a=0$ implies that $q a q=0$, and it is called loyal if it is both left and right loyal.

Note that for a triangular algebra $(\mathcal{A} \oplus \mathcal{B}) \ltimes \mathfrak{X}$, the loyalty of $\mathfrak{X}$ is nothing but the faithfulness of $\mathfrak{X}$ as an $(\mathcal{A}, \mathcal{B})$-module in the sense of Cheung [4]. Combining "the loyalty of $\mathfrak{X}$ " with the current hypotheses of Theorem 2.3 and the equalities $Z(p \mathfrak{A} p)=\pi_{p \mathfrak{A} p}(Z(\mathfrak{A} \ltimes \mathfrak{X})), Z(q \mathfrak{A} q)=\pi_{q \mathfrak{A} q}(Z(\mathfrak{A} \ltimes \mathfrak{X}))$, provide some more sufficient conditions seeking the Lie derivation property for a trivial extension algebra $\mathfrak{A} \ltimes \mathfrak{X}$. In the case where $\mathfrak{X}$ is a loyal $\mathfrak{A}$-module, the existence of an isomorphism between $\pi_{p \mathfrak{A} p}(Z(\mathfrak{A} \ltimes \mathfrak{X}))$ and $\pi_{q \mathfrak{A} q}(Z(\mathfrak{A} \ltimes \mathfrak{X}))$ is the key tool. Indeed, using the same argument as in [4, Proposition 3] (see also [2, Proposition 2.1]), it can be shown that, there exists a unique algebra isomorphism $\tau: \pi_{p \mathfrak{A} p}(Z(\mathfrak{A} \ltimes \mathfrak{X})) \rightarrow \pi_{q \mathfrak{A} q}(Z(\mathfrak{A} \ltimes \mathfrak{X}))$ satisfying papx $=x \tau($ pap $)$ for all $a \in \mathfrak{A}, x \in \mathfrak{X}$.

\section{References}

[1] Bade W.G., Dales H.G., Lykova Z.A., Algebraic and strong splittings of extensions of Banach algebras, Mem. Amer. Math. Soc. 137 (1999), no. 656.

[2] Benkovič D., Lie triple derivations of unital algebras with idempotents, Linear Multilinear Algebra 65 (2015), 141-165.

[3] Cheung W.-S., Mappings on triangular algebras, PhD Dissertation, University of Victoria, 2000.

[4] Cheung W.-S., Lie derivations of triangular algebras, Linear Multilinear Algebra 51 (2003), 299-310.

[5] Du Y., Wang Y., Lie derivations of generalized matrix algebras, Linear Algebra Appl. 437 (2012), 2719-2726.

[6] Ebrahimi Vishki H.R., Mirzavaziri M., Moafian F., Jordan higher derivations on trivial extension algebras, Commun. Korean Math. Soc. 31 (2016), 247-259.

[7] Erfanian Attar A., Ebrahimi Vishki H.R., Jordan derivations on trivial extension algebras, J. Adv. Res. Pure Math. 6 (2014), 24-32.

[8] Ghahramani H., Jordan derivations on trivial extensions, Bull. Iranian Math. Soc. 39 (2013), 635-645.

[9] Ji P., Qi W., Charactrizations of Lie derivations of triangular algebras, Linear Algebra Appl. 435 (2011), 1137-1146.

[10] Martindale III W.S., Lie derivations of primitive rings, Michigan Math. J. 11 (1964), $183-187$.

[11] Moafian F., Higher derivations on trivial extension algebras and triangular algebras, $\mathrm{PhD}$ Thesis, Ferdowsi University of Mashhad, 2015.

[12] Moafian F., Ebrahimi Vishki H.R., Lie higher derivations on triangular algebras revisited, Filomat 30 (2016), no. 12, 3187-3194. 
[13] Mokhtari A.H., Ebrahimi Vishki H.R., More on Lie derivations of generalized matrix algebras, Preprint 2015, arXiv: 1505.02344v1.

[14] Wang Y., Lie n-derivations of unital algebras with idempotents, Linear Algebra Appl. 458 (2014), 512-525.

[15] Zhang Y., Weak amenability of module extensions of Banach algebras, Trans. Amer. Math. Soc. 354 (2002), 4131-4151.

Amir Hosein Mokhtari

Fahimeh Moafian

Department of Pure Mathematics

Ferdowsi University of Mashhad

P.O. Box 1159

MASHHAD 91775

IRAN

e-mail: fahimeh.moafian@yahoo.com

e-mail: amirmkh2002@yahoo.com
Hamid Reza Ebrahimi Vishki

Department of Pure Mathematics

and Center of Excellence in Analysis

on Algebraic Structures (CEAAS)

Ferdowsi University of Mashhad

P.O. Box 1159

MASHHAD 91775

IRAN

e-mail:vishki@um.ac.ir 